\title{
Filtration of Liquid in a Non-isothermal Viscous Porous Medium
}

\author{
Alexander A. Papin* \\ Margarita A. Tokareva ${ }^{\dagger}$ \\ Rudolf A. Virts \\ Altai State University \\ Barnaul, Russian Federation
}

Received 10.08.2020, received in revised form 09.09.2020, accepted 20.10.2020

\begin{abstract}
The solvability of the initial-boundary value problem is proved for the system of equations of one-dimensional unsteady fluid motion in a heat-conducting viscous porous medium.
\end{abstract}

Keywords: Darcy's law, poroelasticity, filtration, solvability, thermal conductivity.

Citation: A.A.Papin, M.A. Tokareva, R.A.Virts, Filtration of Liquid in a Non-isothermal Viscous Porous Medium, J. Sib. Fed. Univ. Math. Phys., 2020, 13(6), 763-773.

DOI: 10.17516/1997-1397-2020-13-6-763-773.

\section{Problem Statement}

The urgency of a theoretical study of filtration problems in porous media is associated with their wide application in solving important practical problems: filtration near river dams, reservoirs and other hydraulic structures; movement of magma in the earth's crust, etc. In many practical problems the porosity of the medium is variable, and the medium is deformed. The model of fluid filtration in a viscous non-isothermal porous medium considered in the work is based on the laws of conservation of masses and energy, Darcy's law, as well as rheological relationships for porosity and pressures. The system of equations has the following form $[1,2]$ :

$$
\begin{gathered}
\frac{\partial(1-\phi) \rho_{s}}{\partial t}+\frac{\partial}{\partial x}\left((1-\phi) \rho_{s} v_{s}\right)=0, \quad \frac{\partial\left(\rho_{f} \phi\right)}{\partial t}+\frac{\partial}{\partial x}\left(\rho_{f} \phi v_{f}\right)=0 \\
\phi\left(v_{f}-v_{s}\right)=-\frac{K(\phi)}{\mu}\left(\frac{\partial p_{f}}{\partial x}-\rho_{f} g\right), \quad \frac{\partial v_{s}}{\partial x}=-\frac{1}{\xi(\phi, \theta)} p_{e} \\
\frac{\partial p_{t o t}}{\partial x}=-\rho_{t o t} g, \quad \rho_{t o t}=\phi \rho_{f}+(1-\phi) \rho_{s}, \quad p_{e}=p_{t o t}-p_{f}, \quad p_{t o t}=\phi p_{f}+(1-\phi) p_{s} \\
\left(\rho_{f} c_{f} \phi+\rho_{s} c_{s}(1-\phi)\right) \frac{\partial \theta}{\partial t}+\left(\rho_{f} c_{f} \phi v_{f}+\rho_{s} c_{s}(1-\phi) v_{s}\right) \frac{\partial \theta}{\partial x}=\frac{\partial}{\partial x}\left(\lambda \frac{\partial \theta}{\partial x}\right),
\end{gathered}
$$

and is solved in the domain $(x, t) \in Q_{T}=\Omega \times(0, T), \Omega=(0,1)$, under the boundary and initial conditions

\footnotetext{
*papin@math.asu.ru https://orcid.org/0000-0001-7510-9164

†tma25@mail.ru https://orcid.org/0000-0002-7162-342X



(c) Siberian Federal University. All rights reserved
} 


$$
\left.v_{s}\right|_{x=0, x=1}=\left.v_{f}\right|_{x=0, x=1}=\left.\frac{\partial \theta}{\partial x}\right|_{x=0, x=1}=0,\left.\quad \phi\right|_{t=0}=\phi^{0}(x),\left.\quad \theta\right|_{t=0}=\theta^{0}(x) .
$$

This initial-boundary value problem describes the one-dimensional motion of a two-phase medium between impenetrable heat-insulated walls $[1,2]$. Here $\rho_{s}, \rho_{f}, v_{s}, v_{f}$, are, respectively, the constant real densities and velocities of phases ( $s$ is solid porous medium, $f$ is liquid), $\phi$ is porosity (fraction of pores), $p_{s}$ and $p_{f}$ are pressures in solid and liquid phases, $p_{t o t}$ is total medium pressure, $p_{e}$ is effective pressure, $\rho_{t o t}$ is two-phase density, $\theta$ is absolute temperature, $g$ isdensity of the mass forces, $c_{s}$ and $c_{f}$ are heat capacities for at constant volume of phases, $K(\phi)$ is permeability coefficient, $\mu$ is dynamic fluid viscosity, $\xi(\phi, \theta)$ is bulk viscosity coefficient, $\lambda(\phi)$ is heat conductivity coefficient (the prescribed functions). The problem is written in Euler coordinates $(x, t)$.

For the permeability coefficient $K(\phi)$, a well-known dependence of the form is used $K(\phi)=$ $K^{\prime} \phi^{n}$, where $K^{\prime}=$ const $>0, n=3[1]$. The bulk viscosity coefficient $\xi(\phi, \theta)$ is usually taken as $\xi(\phi, \theta)=\eta(\theta) / \phi^{m}, m \in[0,2]$, where $\eta(\theta)$ is the coefficient of dynamic viscosity of the skeleton, which characterizes the relationship between the strain rate tensor and the stress tensor and is determined from the experiment under uniaxial compression [3,4]. The following dependence is taken as a model one: $\eta(\theta)=\eta_{r} \exp \left(Q_{r}\left(1-\theta / \theta_{r}\right) / R \theta\right), \eta_{r}, Q_{r}, \theta_{r}, R$ are positive constants (analog of the Arrhenius formula for the dependence of the reaction rate on temperature) [1]. The thermal conductivity coefficient of the medium $\lambda(\phi)$ is taken in the form $\lambda(\phi)=\lambda_{f} \phi+\lambda_{s}(1-\phi)$, where $\lambda_{f}, \lambda_{s}$ are the thermal conductivity of liquid and solid phase (averaged thermal conductivity) [2] In what follows, the notations are used $k(\phi)=K(\phi) / \mu, 1 / \xi(\phi, \theta)=a_{1}(\phi) \xi_{1}(\theta), a_{1}(\phi)=\phi^{m}$, $\xi_{1}(\theta)=1 / \eta(\theta)$.

The local in time solvability of the initial-boundary value problem for the equations (1)-(3) at constant temperature in the case of a compressible fluid was established in the work [5]. A numerical analysis of the initial-boundary value problem for the system (1)-(3) is carried out in [6]: difference schemes are constructed and their convergence is established. In paper [7], the global solvability of the problem (1)- (3) is proved in the case of constant phase densities.

Systems of equations similar in structure were considered in [8-16]. The local solvability of the Cauchy problem in Sobolev spaces was established in [8]. The simplest models of deformation of a poroelastic medium were studied in $[9,10]$. Self-similar solutions of the traveling wave type for the equations of magma motion were considered in $[11,12]$. The works $[14,15]$ are devoted to numerical calculations. The problem of substantiating multidimensional models of fluid filtration in poroelastic media is open.

In the notation of function spaces, we follow [15]: $C^{l+\alpha, r+\beta}\left(Q_{T}\right)$ is the Hölder space, where $l, r$ are natural numbers, $(\alpha, \beta) \in(0,1]$, with the norm $\|f\|_{C^{l+\alpha, r+\beta}\left(Q_{T}\right)}$.

In this paper, we prove the local classical solvability of the problem (1)-(4) in the case when the bulk viscosity coefficient $\xi$ is a function of porosity and temperature. An example of decidability "in the whole" is given.

Definition. By a solution of problem (1)-(5) we mean the set of functions $\phi, \phi_{t}, \theta, v_{s}, v_{f} \in$ $C^{2+\alpha, 1+\beta}\left(Q_{T}\right), p_{f}, p_{s} \in C^{1+\alpha, 1+\beta}\left(Q_{T}\right)$, such that $0<\phi<1,0<\theta<\infty$. These functions satisfy the equations (1)-(4) and the initial and boundary conditions (5) and regarded as continuous functions in $Q_{T}$.

Theorem 1. Suppose that the data of problem (1)-(5) satisfies the following conditions:

1) the functions $k(\phi), a_{1}(\phi), \lambda(\phi), \xi_{1}(\theta)$ and their derivatives up to the second order are continuous for $\phi \in(0,1), \theta \in(0, \infty)$ and satisfy the conditions

$$
k_{0}^{-1} \phi^{q_{1}}(1-\phi)^{q_{2}} \leqslant k(\phi) \leqslant k_{0} \phi^{q_{3}}(1-\phi)^{q_{4}},
$$




$$
\begin{gathered}
k_{0}^{-1} \phi^{q_{5}}(1-\phi)^{q_{6}} \leqslant \lambda(\phi) \leqslant k_{0} \phi^{q_{7}}(1-\phi)^{q_{8}}, \quad \xi_{1}(\theta)>0, \quad \theta \in(0, \infty), \\
\frac{1}{\xi(\phi)}=a_{0}(\phi) \phi^{\alpha_{1}}(1-\phi)^{\alpha_{2}-1}, \quad 0<R_{1} \leqslant a_{0}(\phi) \leqslant R_{2}<\infty
\end{gathered}
$$

where $k_{0}, \alpha_{i}, R_{i}, i=1,2$ are positive constants, $q_{1}, \ldots, q_{8}$ are fixed real numbers.

2) the function $g$, the initial functions $\phi^{0}$ and $\theta^{0}$ satisfy the following smoothness conditions:

$$
g \in C^{1+\alpha, 1+\beta}\left(\bar{Q}_{T}\right), \quad \theta^{0}, \phi^{0} \in C^{2+\alpha}(\bar{\Omega}),
$$

and the inequalities

$0<m_{0} \leqslant \phi^{0}(x) \leqslant M_{0}<1, \quad 0<m \leqslant \theta^{0}(x) \leqslant M<\infty, \quad|g(x, t)| \leqslant g_{0}<\infty, \quad x \in \bar{\Omega}, \quad t \in(0, T)$, where $m_{0}, M_{0}, m, M, g_{0}$ are given positive constants.

Then problem (1)-(5) has a local solution, i.e., there exists a value of $t_{0}$ such that $\phi(x, t), \phi_{t}(x, t), \theta(x, t) \in C^{2+\alpha, 1+\beta}\left(\bar{Q}_{t_{0}}\right), \quad\left(v_{s}(x, t), v_{f}(x, t)\right) \in C^{2+\alpha, \beta}\left(\bar{Q}_{t_{0}}\right), \quad\left(p_{f}(x, t), p_{s}(x, t)\right) \in$ $C^{1+\alpha, \beta}\left(\bar{Q}_{t_{0}}\right)$.

Moreover, $0<\phi(x, t)<1,0<\theta(x, t)<\infty$ in $\bar{Q}_{t_{0}}$.

Theorem 2. Let, in addition to the conditions of Theorem 1, the functions $k(\phi), \xi(\phi, \theta)$ satisfy the conditions

$$
k(\phi)=\frac{K}{\mu}, \quad \xi(\phi, \theta)=\frac{\eta(\theta)}{\phi},
$$

where $K, \mu$ are positive constants.

Then for all $t \in[0, T], T<\infty$ uniqueness solution of problem (1)-(5) exists, and there are numbers $0<m_{1}<M_{1}<1,0<m_{2}<M_{2}$ such that $m_{1} \leqslant \phi(x, t) \leqslant M_{1}, m_{2} \leqslant \theta(x, t) \leqslant M_{2}$, $(x, t) \in Q_{T}$.

\section{Local solvability}

Proof of Theorem 1. When proving Theorems 1 and 2, it is convenient to use the Lagrange variables [17]. Suppose that $\bar{x}=\bar{x}(\tau, x, t)$ is a solution of the Cauchy problem

$$
\frac{\partial \bar{x}}{\partial \tau}=v_{s}(\bar{x}, \tau),\left.\quad \bar{x}\right|_{\tau=t}=x
$$

We set $\hat{x}=\bar{x}(0, x, t)$ and take $\hat{x}$ and $t$ for the new variables. Then $\hat{J}(\hat{x}, t)=\frac{\partial \hat{x}}{\partial x}(x, t)=$ $=(1-\phi(\hat{x}, t)) /\left(1-\phi^{0}(\hat{x})\right)$ is the Jacobian of the transformation. Following [5], we rewrite the system $(1)-(4)$ :

$$
\begin{gathered}
\frac{\partial}{\partial t}\left(\frac{\phi}{1-\phi}\right)=\frac{\partial}{\partial x}\left(k(\phi)(1-\phi) \frac{\partial}{\partial x}\left(\frac{1}{\xi_{1}(\theta)} \frac{\partial G(\phi)}{\partial t}\right)-k(\phi) g\left(\rho_{t o t}+\rho_{f}\right)\right) \\
\left.\left((1-\phi) \frac{\partial}{\partial x}\left(\frac{1}{\xi_{1}(\theta)} \frac{\partial G}{\partial t}\right)-g\left(\rho_{t o t}+\rho_{f}\right)\right)\right|_{x=0, x=1}=0,\left.\quad \phi\right|_{t=0}=\phi^{0}(x) \\
\left(c_{s} \rho_{s}+c_{f} \rho_{f} \frac{\phi}{1-\phi}\right) \frac{\partial \theta}{\partial t}+c_{f} \rho_{f} \phi\left(v_{f}-v_{s}\right) \frac{\partial \theta}{\partial x}=\frac{\partial}{\partial x}\left(\lambda(1-\phi) \frac{\partial \theta}{\partial x}\right) \\
\left.\frac{\partial \theta}{\partial x}\right|_{x=0, x=1}=0,\left.\quad \theta\right|_{t=0}=\theta^{0}(x) \\
\frac{\partial G(\phi)}{\partial t}=\xi_{1}(\theta) p_{e}, \quad \frac{d G}{d \phi}=\frac{1}{a_{1}(\phi)(1-\phi)}
\end{gathered}
$$


In the system (6)-(10), the basic equations are (6) and (8) for the required functions $\phi$ and $\theta$.

We substitute in the coefficients of the equation (6) and the boundary condition (7) instead of $\theta(x, t)$ an arbitrary smooth function $\theta_{0}(x, t) \in C^{2+\alpha_{1}, 1+\beta_{1}}\left(Q_{T}\right)$, which satisfies the inequalities $0<m \leqslant \theta^{0}(x) \leqslant M<\infty$. We retain the previous notation $\phi$ for solving the arising problem and the latter is called Problem I.

Lemma 1. Let the data of problem I satisfy the conditions of the theorem. Then problem I has a unique local solution, i.e., there exists a value of $t_{0}$ such that

$$
\left(\phi, \phi_{t}\right) \in C^{2+\alpha, 1+\beta}\left(Q_{t_{0}}\right), \phi \in(0,1) .
$$

Proof. Suppose that $z=\frac{1}{\xi_{1}\left(\theta_{0}\right)} \frac{\partial G}{\partial t}$, we arrive at the following problem for $G, z$ :

$$
\begin{gathered}
z=\frac{1}{\xi_{1}\left(\theta_{0}\right)} \frac{\partial G}{\partial t},\left.\quad G\right|_{t=0}=G\left(\phi^{0}\right)=G^{0}(x) \\
\frac{z}{d\left(G, \theta_{0}\right)}-\frac{\partial}{\partial x}\left(a(G) \frac{\partial z}{\partial x}-b(G)\right)=0,\left.\quad\left(a(G) \frac{\partial z}{\partial x}-b(G)\right)\right|_{x=0, x=1}=0
\end{gathered}
$$

where

$$
\left.\left.d\left(G, \theta_{0}\right)\right)\right)=\frac{1-\phi(G)}{a_{1}(\phi(G)) \xi_{1}\left(\theta_{0}\right)}, \quad a(G)=k(\phi(G))(1-\phi(G)), \quad b(G)=k(\phi(G)) g\left(\rho_{\text {tot }}+\rho_{f}\right) .
$$

Since $0<m_{0} \leqslant \phi^{0}(x) \leqslant M_{0}<1$ and the function $G(\phi)$ is monotone, then $G\left(m_{0}\right) \leqslant G^{0}(x) \leqslant$ $G\left(M_{0}\right)$. From $(11)$ when the inequality $\max _{(x, t)}\left|\xi_{1}(\theta) z(x, t)\right| \leqslant c_{0}$ we have that there is a value $t_{0}$, such that for all $t \leqslant t_{0}$ the estimates take place

$$
\begin{gathered}
G_{1}\left(m_{0}\right)=G\left(m_{0}\right)-c_{0} t_{0} \leqslant G(x, t) \leqslant G\left(M_{0}\right)+c_{0} t_{0}=G_{2}\left(M_{0}\right), \\
0 \leqslant G^{-1}\left(G_{1}\left(m_{0}\right)\right) \leqslant \phi(x, t) \leqslant G^{-1}\left(G_{2}\left(M_{0}\right)\right)<1 .
\end{gathered}
$$

Let $G_{0}(x, t)$ be a function continuous in $x$ and $t$, satisfying inequalities (13) and having a continuous derivative $\partial G_{0} / \partial x$ with respect to $x, t$. Substituting $G_{0}(x, t)$ instead of $G(x, t)$ into the coefficients of the equation (12) and the boundary conditions, we arrive at a linear problem for $z$, in which $a>0, b>0$ and $d>0$. The solution to this problem is unique. Existence follows, for example, from Hilbert's theorem [18] for ordinary linear equations of the second order. The $t$ variable plays the role of a parameter. Thus, $\left(z, z_{x}, z_{x x}\right) \in C\left(Q_{t_{0}}\right)$. After finding $z(x, t)$, we can find a new value $G(x, t)$ from the equation (11). This value will satisfy the condition (13).

To prove the solvability of problem I, we use the method of successive approximations. Let $z^{i}(x, t)$ and $G^{i}(x, t)$ be a solution to the problem

$$
\begin{gathered}
\frac{\partial G^{i+1}}{\partial t}=\xi_{1}\left(\theta_{0}\right) z^{i+1}, \quad G^{i+1}(x, 0)=G^{0}(x), \\
\frac{z^{i+1}}{d\left(G^{i}\right)}-\frac{\partial}{\partial x}\left(a\left(G^{i}\right) \frac{\partial z^{i+1}}{\partial x}-b\left(G^{i}\right)\right)=0, \\
\left.\left(a\left(G^{i}\right) \frac{\partial z^{i+1}}{\partial x}-b\left(G^{i}\right)\right)\right|_{x=0, x=1}=0
\end{gathered}
$$

where $i=0,1,2, \ldots$ Substituting $G^{0}(x)$ into the equation for $z$ at the first step, we find $z^{1}(x, t)$. After that, from the equation for $G$ we find $G^{1}(x, t)$, etc. For each $i$ there is a unique solution 
$z^{i}(x, t)$ and $G^{i}(x, t)$, satisfying (13). It is checked in a standard way that for a small value of $t_{0}$ the solutions $z^{i}(x, t), G^{i}(x, t)$ and their derivatives up to the second order inclusive are bounded uniformly in $i$.

We put $y^{i+1}=z^{i+1}-z^{i}, \omega^{i+1}=G^{i+1}-G^{i}$. We have

$$
\begin{gathered}
\frac{\partial \omega^{i+1}}{\partial t}=\xi_{1}\left(\theta_{0}\right) y^{i+1},\left.\quad \omega^{i+1}\right|_{t=0}=0 \\
\frac{y^{i+1}}{d\left(G^{i}\right)}+A_{1} \omega^{i}-\frac{\partial}{\partial x}\left(a y_{x}^{i+1}+A_{2} \omega^{i}\right)=0 \\
\left.\left(a y_{x}^{i+1}+A_{2} \omega^{i}\right)\right|_{x=0, x=1}=0
\end{gathered}
$$

where the coefficients $A_{1}, A_{2}$ are easily recovered and are limited. We have from this system the following inequalities

$$
\begin{gathered}
\int_{0}^{1}\left(\left|y^{i+1}\right|^{2}+\left|y_{x}^{i+1}\right|^{2}\right) d x \leqslant c_{1} \int_{0}^{1}\left|\omega^{i}\right|^{2} d x \leqslant c_{1} \max _{x}\left|\omega^{i}\right|^{2} \\
\max _{x}\left|\omega^{i+1}\right| \leqslant c_{1} \int_{0}^{t} \max _{x}\left|y^{i+1}\right| d \tau
\end{gathered}
$$

where the constant $c_{1}$ does not depend on $i$. Taking into account the last inequality for the function $v^{i}(t)=\max _{x}\left|y^{i}(x, t)\right|^{2}$ we get $v^{i+1}(t) \leqslant c_{2} \int_{0}^{t} v^{i}(\tau) d \tau$ and therefore [19], $v^{i}(t) \leqslant$ $\left(c_{2} T\right)^{i} v^{0} / i$ ! $\rightarrow 0$ for $i \rightarrow \infty$. After that it is easy to establish that the sequences $z^{i}, G^{i}$ are fundamental in $C\left(Q_{t_{0}}\right)$ and have limits $z(x, t) \in C\left(Q_{t_{0}}\right)$ and $G(x, t) \in C\left(Q_{t_{0}}\right)$. The sequences $z_{x}^{i}, z_{x x}^{i}, G_{t}^{i}$ are also fundamental. Passing to the limit as $i \rightarrow \infty$, we obtain that the limit functions satisfy the problem (11), (12). The uniqueness of the solution is proved similarly to [7]. Increasing the smoothness of the initial data to those specified in the conditions of Theorem 1 allows us to obtain that $\phi(x, t), \phi_{t}(x, t) \in C^{2+\alpha, 1+\beta}\left(\bar{Q}_{t_{0}}\right)$.

Lemma 1 is proved.

Substituting $\theta_{0}(x, t)$ and the solution to Problem I into the coefficients of equation (8), we arrive at a linear problem for $\theta(x, t)$ of the form

$$
\begin{gathered}
Q \frac{\partial \theta}{\partial t}+V \frac{\partial \theta}{\partial x}=\frac{\partial}{\partial x}\left(\lambda(1-\phi) \frac{\partial \theta}{\partial x}\right), \\
\left.\frac{\partial \theta}{\partial x}\right|_{x=0, x=1}=0,\left.\quad \theta\right|_{t=0}=\theta^{0}(x),
\end{gathered}
$$

where

$$
Q=\rho_{s} c_{s}+\rho_{f} c_{f} \frac{\phi}{1-\phi}, \quad V=c_{f} \rho_{f} \phi\left(v_{f}-v_{s}\right)=\rho_{f} c_{f} k(\phi)\left((1-\phi) \frac{\partial z}{\partial x}+g\left(\rho_{t o t}+\rho_{f}\right)\right) .
$$

The unique solvability of this problem in Holder classes follows from [19], and the solution satisfies the estimate

$$
0<\underline{\theta}=\min _{x} \theta^{0}(x) \leqslant \theta(x, t) \leqslant \max _{x} \theta^{0}(x)=\bar{\theta}<\infty .
$$

After these remarks, the local solvability of the problem (6)-(9) can easily be obtained using the Schauder theorem according to the scheme used in [7]. 
After finding $\phi, \theta$, the remaining functions from the system (1)-(4) can be defined as follows. We find the phase velocities from (1)

$$
\begin{gathered}
v_{f}(x, t)=-\frac{1}{\phi} \int_{0}^{x} \frac{\partial \phi}{\partial t} d \xi \in C^{2+\alpha, \beta}\left(Q_{t_{0}}\right) \\
v_{s}(x, t)=-\frac{1}{1-\phi} \int_{0}^{x} \frac{\partial(1-\phi)}{\partial t} d \xi \in C^{2+\alpha, \beta}\left(Q_{t_{0}}\right) .
\end{gathered}
$$

From $(3)$ we find $p_{t o t}(x, t)=p^{0}(t)-\int_{0}^{x} \rho_{t o t} g d \xi \in C^{3+\alpha, 1+\beta}\left(Q_{t_{0}}\right)$.

From (2) we have $p_{e}(x, t)=-\frac{\partial v_{s}}{\partial x} \xi(\phi, \theta) \in C^{1+\alpha, \beta}\left(Q_{t_{0}}\right)$, then

$$
p_{f}(x, t)=p_{t o t}-p_{e} \in C^{1+\alpha, \beta}\left(Q_{t_{0}}\right), \quad p_{s}(x, t)=\frac{p_{t o t}}{1-\phi}-\frac{\phi}{1-\phi} p_{f} \in C^{1+\alpha, \beta}\left(Q_{t_{0}}\right) .
$$

Theorem 1 is proved.

\section{Global solvability}

Proof of Theorem 2. By Theorem 1, we will assume that on the interval $\left[0, t_{0}\right]$ there exists a solution to the problem (1)-(5), and $0<\phi(x, t)<1,0<\theta(x, t)<\infty, x \in \Omega, t \in\left[0, t_{0}\right]$. After obtaining the necessary a priori estimates that do not depend on the value of $t_{0}$, the local solution can be continued to the entire segment $[0, T]$.

Lemma 2. Under the conditions of Theorem 2, for all $t \in[0, T]$ the following relations hold:

$$
\begin{gathered}
\int_{0}^{1} s(x, t) d x=\int_{0}^{1} s^{0}(x) d x, \quad s=\frac{\phi}{1-\phi}, \quad s^{0}=s(x, 0), \\
0<\underline{\theta} \equiv \min _{x \in[0,1]} \theta^{0}(x) \leqslant \theta(x, t) \leqslant \max _{x \in[0,1]} \theta^{0}(x) \equiv \bar{\theta}<\infty, \\
\int_{0}^{1} \frac{1}{\xi_{1}(\theta)} \frac{a_{1}}{1-\phi}\left(\frac{\partial G}{\partial t}\right)^{2} d x+\frac{1}{2} \int_{0}^{1} k(\phi)(1-\phi)\left|\frac{\partial}{\partial x}\left(\frac{1}{\xi_{1}(\theta)} \frac{\partial G}{\partial t}\right)\right|^{2} d x \leqslant \\
\leqslant \frac{1}{2} \int_{0}^{1} \frac{k(\phi)}{1-\phi} g^{2}\left(\rho_{\text {tot }}+\rho_{f}\right)^{2} d x \leqslant N .
\end{gathered}
$$

Hereinafter, $N$ denotes a constant that depends only on the data of the problem (1)-(5) and does not depend on $t_{0}$.

Proof. Let us integrate the equation (6) over $x$ from 0 to 1 and take into account the boundary condition (7). After integration over time from 0 to the current value of $t$, we arrive at the equality (14).

The equation (8) is written in a divergent form:

$$
\begin{gathered}
\frac{\partial}{\partial t}\left(\theta\left(c_{s} \rho_{s}+c_{f} \rho_{f} \frac{\phi}{1-\phi}\right)\right)+\frac{\partial}{\partial x}\left(\theta c_{f} \rho_{f} \phi\left(v_{f}-v_{s}\right)-\lambda(1-\phi) \frac{\partial \theta}{\partial x}\right)= \\
=\theta\left[\frac{\partial}{\partial t}\left(c_{s} \rho_{s}+c_{f} \rho_{f} \frac{\phi}{1-\phi}\right)+\frac{\partial}{\partial x}\left(c_{f} \rho_{f} \phi\left(v_{f}-v_{s}\right)\right)\right] . \\
-768-
\end{gathered}
$$


The right-hand side of this equality is equal to zero, since the second equation from (1) in Lagrange variables becomes [5]

$$
\frac{\partial}{\partial t}\left(\frac{\phi}{1-\phi}\right)+\frac{\partial}{\partial x}\left(\phi\left(v_{f}-v_{s}\right)\right)=0
$$

In particular, from (17) we have

$$
\int_{0}^{1}\left(c_{f} \rho_{f} \frac{\phi}{1-\phi}+c_{s} \rho_{s}\right) \theta d x=\int_{0}^{1}\left(c_{f} \rho_{f} \frac{\phi^{0}}{1-\phi^{0}}+c_{s} \rho_{s}\right) \theta^{0} d x
$$

and therefore $\theta(x, t) \in L_{1}[0,1]$ for all $t \in[0, T]$.

Let the smooth function $\kappa(\theta)$ satisfy the condition $\kappa^{\prime \prime}(\theta)=d^{2} \kappa / d \theta^{2} \geqslant 0$. Multiplying the equation (8) by $\kappa^{\prime}(\theta)=d \kappa / d \theta$, and following the equality (17) we reduce the resulting equality to the form

$$
\begin{array}{r}
\frac{\partial}{\partial t}\left(\left(c_{s} \rho_{s}+c_{f} \rho_{f} \frac{\phi}{1-\phi}\right) \kappa(\theta)\right)+\frac{\partial}{\partial x}\left(c_{f} \rho_{f} \phi\left(v_{f}-v_{s}\right) \kappa(\theta)\right)= \\
=\frac{\partial}{\partial x}\left(\lambda(1-\phi) \frac{\partial \kappa(\theta)}{\partial x}\right)-\kappa^{\prime \prime}(\theta)\left(\frac{\partial \theta}{\partial x}\right)^{2} \lambda(1-\phi)
\end{array}
$$

In the case $\kappa(\theta)=\theta^{p}, p>1$, from (18) we deduce

$$
\int_{0}^{1} \theta^{p}(x, t) d x \leqslant \max _{x \in[0,1]}\left(\frac{c_{f} \rho_{f}}{c_{s} \rho_{s}} \frac{\phi^{0}(x)}{1-\phi^{0}(x)}+1\right) \int_{0}^{1}\left|\theta^{0}(x)\right|^{p} d x .
$$

Whence, in the standard way, we get that $\theta(x, t) \leqslant \max _{x \in[0,1]} \theta^{0}(x)$ for all $t \in[0, T], x \in[0,1]$. Put $\theta_{1}=1 / \theta$ and the equation (6) can be represented as ${ }^{6}$

$$
\left(c_{s} \rho_{s}+c_{f} \rho_{f} \frac{\phi}{1-\phi}\right) \frac{\partial \theta_{1}}{\partial t}+c_{f} \rho_{f}\left(v_{f}-v_{s}\right) \frac{\partial \theta_{1}}{\partial x}=\frac{\partial}{\partial x}\left(\lambda(1-\phi) \frac{\partial \theta_{1}}{\partial x}\right)-2 \lambda(1-\phi)\left(\frac{\partial \theta_{1}}{\partial x}\right)^{2} \theta .
$$

Multiplying (8) by $\kappa_{1}^{\prime}\left(\theta_{1}\right)=d \kappa_{1} / d \theta_{1}, \kappa_{1}=\theta_{1}^{p}$, and integrating over $x$, we arrive at a relation of the form (14) for $\theta_{1}(x, t)$. Therefore $\theta(x, t) \geqslant \min _{x \in[0,1]} \theta^{0}(x)$ for all $t \in[0, T], x \in[0,1]$.

Multiplying the equation (6) by $\frac{1}{\xi_{1}(\theta)} \frac{\partial G}{\partial t}$ and integrating over $x$ we arrive at the relation

$$
\begin{gathered}
\int_{0}^{1} \frac{1}{\xi_{1}(\theta)} \frac{a_{1}(\phi)}{1-\phi}\left(\frac{\partial G}{\partial t}\right)^{2} d x+\int_{0}^{1} k(\phi)(1-\phi)\left|\frac{\partial}{\partial x}\left(\frac{1}{\xi_{1}(\theta)} \frac{\partial G}{\partial t}\right)\right| d x= \\
=\int_{0}^{1} k(\phi) g\left(\rho_{t o t}+\rho_{f}\right) \frac{\partial}{\partial x}\left(\frac{1}{\xi_{1}(\theta)} \frac{\partial G}{\partial t}\right) d x \leqslant \\
\leqslant \frac{1}{2} \int_{0}^{1} k(\phi)(1-\phi)\left|\frac{\partial}{\partial x}\left(\frac{1}{\xi_{1}(\theta)} \frac{\partial G}{\partial t}\right)\right|^{2} d x+\frac{1}{2} \int_{0}^{1} \frac{k(\phi)}{1-\phi} g^{2}\left(\rho_{t o t}+\rho_{f}\right)^{2} d x .
\end{gathered}
$$

The last term on the right-hand side is bounded uniformly in $t_{0}$, since $\phi<1$ and, therefore, $\rho_{\text {tot }} \leqslant \max \left(\rho_{f}, \rho_{s}\right)$. Finally, due to $(14)$ we have

$$
\int_{0}^{1} \frac{d x}{1-\phi}=1+\int_{0}^{1} s^{0}(x) d x .
$$

Lemma 2 is proved. 
Lemma 3. Under the conditions of Theorem 2, for all $t \in[0, T], x \in[0,1]$ the estimate takes place

$$
0<m \leqslant \phi(x, t) \leqslant M<1 .
$$

Proof. From the inequality (16) by the conditions of Theorem 2 it follows

$$
\int_{0}^{1}\left|\frac{\partial}{\partial x}\left(\frac{1}{\xi_{1}(\theta)} \frac{\partial G}{\partial t}\right)\right| d x \leqslant\left(\int_{0}^{1} \frac{d x}{1-\phi}\right)^{1 / 2}\left(\int_{0}^{1}(1-\phi)\left|\frac{\partial}{\partial x}\left(\frac{1}{\xi_{1}(\theta)} \frac{\partial G}{\partial t}\right)\right|^{2} d x\right)^{1 / 2} .
$$

From (6) it also follows that

$$
\int_{0}^{1} \frac{a_{1}}{1-\phi} \frac{\partial G}{\partial t} d x=0
$$

and, therefore, there is a point $x_{0}(t)$ at which $\frac{\partial G}{\partial t}\left(x_{0}(t), t\right)=0$. Therefore

$$
\min _{x \in(0,1)}\left|\frac{1}{\xi_{1}(\theta)}\right|\left|\frac{\partial G}{\partial t}\right| \leqslant\left|\frac{1}{\xi_{1}(\theta)} \frac{\partial G}{\partial t}\right| \leqslant \int_{0}^{1}\left|\frac{\partial}{\partial x}\left(\frac{1}{\xi_{1}(\theta)} \frac{\partial G}{\partial t}\right)\right| d x \leqslant N .
$$

Taking into account (15) and the conditions of Theorem 2, from the last inequality we have

$$
|\ln s(x, t)| \leqslant|G(x, t)| \leqslant\left|G^{0}(x)\right|+N_{1} T \leqslant N_{2} .
$$

Then we arrive at (19) with $m=\left(1+e^{N_{2}}\right)^{-1}, M=\left(1+e^{-N_{2}}\right)^{-1}$.

Let $z=\frac{1}{\xi_{1}(\theta)} \frac{\partial G}{\partial t}$. The problem $(6),(7)$ takes the form

$$
\begin{gathered}
\frac{a_{1}(\phi) \xi_{1}(\theta) z}{(1-\phi)}=\frac{\partial}{\partial x}\left(k(\phi)(1-\phi) \frac{\partial z}{\partial x}-k(\phi) g\left(\rho_{t o t}+\rho_{f}\right)\right) \\
\left.\left(k(\phi)(1-\phi) \frac{\partial z}{\partial x}-k(\phi) g\left(\rho_{t o t}+\rho_{f}\right)\right)\right|_{x=0, x=1}=0 .
\end{gathered}
$$

By Lemmas 2 and 3, we have

$$
\int_{0}^{t} \int_{0}^{1} \theta_{x}^{2} d x d \tau+\int_{0}^{1}\left(z^{2}+z_{x}^{2}+\theta_{x}^{2}\right) d x \leqslant N_{3}
$$

where $N_{3}$ is a positive constant depending on the initial data, parameters and problem constants, but does not depend on $t_{0}$.

Using the representation

$$
G(\phi)=\int_{0}^{t} \xi_{1}(\theta) z d \tau+G\left(\phi^{0}\right)
$$

we get

$$
G^{\prime}(\phi) \phi_{x}=\int_{0}^{t}\left(z_{x} \xi_{1}(\theta)+z \xi_{1}^{\prime} \theta_{x}\right) d \tau+G_{x}\left(\phi^{0}\right)
$$

Therefore

$$
\int_{0}^{1} \phi_{x}^{2} d x \leqslant N_{4}
$$

The equation for function $z(x, t)$ takes form

$$
a_{0}(\phi, \theta) z=a_{1}(\phi) z_{x x}+a_{1}^{\prime}(\phi) \phi_{x} z_{x}+a_{2}^{\prime}(\phi) \phi_{x} .
$$


The coefficients $a_{0}(\phi, \theta)>0, a_{1}(\phi)>0, a_{2}(\phi)$ are limited and easy to calculate.

We have

$$
\int_{0}^{1} z_{x x}^{2} d x \leqslant C_{1}\left(\int_{0}^{1}\left(z^{2}+\phi_{x}^{2}\right) d x+\int_{0}^{1}\left|z_{x x} z_{x} \phi_{x}\right| d x\right)
$$

where

$$
\begin{gathered}
I_{1}=\int_{0}^{1}\left|z_{x x}\right|\left|z_{x} \phi_{x}\right| d x \leqslant \max \left|z_{x}\right|\left(\int_{0}^{1} z_{x x}^{2} d x\right)^{1 / 2}\left(\int_{0}^{1} \phi_{x}^{2} d x\right)^{1 / 2} \leqslant \\
\leqslant C_{1}\left(\left(\int_{0}^{1} z_{x x}^{2} d x\right)^{1 / 2}\left(\int_{0}^{1} \phi_{x} d x\right)^{1 / 2}+\left(\int_{0}^{1} z_{x x}^{2} d x\right)^{3 / 4}\left(\int_{0}^{1} \phi_{x} d x\right)^{1 / 2}\right) .
\end{gathered}
$$

The constant $C_{1}$ is not depend on $t_{0}$.

Therefore

$$
\max _{x}\left|z_{x}\right|+\int_{0}^{1} z_{x x}^{2} d x \leqslant N_{4}
$$

The equation for the function $\theta(x, t)$ has the form

$$
\theta_{t}+a_{3}\left(\phi, z_{x}\right) \theta_{x}=a_{4}(\phi) \theta_{x x}+a_{5}(\phi) \phi_{x} \theta_{x}
$$

where the coefficients $a_{4}(\phi)>0, a_{3}\left(\phi, z_{x}\right), a_{5}(\phi)$ are limited and easy to calculate.

Since

$$
\begin{aligned}
\int_{0}^{1}\left|\theta_{x} \theta_{x x} \phi_{x}\right| d x & \leqslant \max _{x}\left|\theta_{x}\right|\left(\int_{0}^{1} \theta_{x x}^{2} d x\right)^{1 / 2}\left(\int_{0}^{1} \phi_{x}^{2} d x\right)^{1 / 2} \leqslant \\
& \leqslant c\left(\int_{0}^{1} \theta_{x x}^{2} d x\right)^{3 / 4}\left(\int_{0}^{1} \phi_{x}^{2} d x\right)^{1 / 2}\left(\int_{0}^{1} \theta_{x}^{2} d x\right)^{1 / 4}
\end{aligned}
$$

then from the equation for $\theta$ we have

$$
\int_{0}^{1} \theta_{x}^{2} d x+\int_{0}^{t} \int_{0}^{1}\left(\theta_{t}^{2}+\theta_{x x}^{2}\right) d x d \tau \leqslant N_{5}
$$

To complete the proof of Theorem 2, it is necessary to obtain the Holder continuity in $x, t$ of the functions $\phi_{x}$ and $z_{x}$ included in the coefficients of the equations for $z$ and $\theta$. From the embedding $z_{x x} \in L_{2}[0,1]$ and the representation for $\phi$ we have $\phi_{x x} \in L_{2}[0,1]$. Then for $w=\theta_{x}$ we get

$$
\int_{0}^{1}\left(\theta_{t}^{2}+w_{x}^{2}\right) d x+\int_{0}^{t} \int_{0}^{1}\left(w_{t}^{2}+w_{x x}^{2}\right) d x d \tau \leqslant N_{6} .
$$

After that we deduce that $\left|\phi_{x t}\right| \leqslant N_{7}$. Finally, following [7] for the function $\sigma=z_{t}$ we get $\sigma_{x} \in L_{2}[0,1]$.

Theorem 2 is proved.

\section{Conclusion}

The local solvability in the Holder classes of the initial-boundary value problem of onedimensional fluid motion in a nonisothermal viscous porous medium is proved. An example of decidability is given at any finite time interval.

The work was carried out in accordance with the State Assignment of the Russian Ministry of Science and Higher Education entitled 'Modern methods of hydrodynamics for environmental management, industrial systems and polar mechanics' (Govt. contract code: FZMW-2020-0008, 24 January 2020). 


\section{References}

[1] J.A.D.Connoly, Y.Y.Podladchikov, Compaction-driven fluid flow in viscoelastic rock, Geodinamica Acta, 11(1998), no 2-3, 55-84.

[2] A.Fowler, Mathematical Geoscience, Springer-Verlag London Limited, 2011.

[3] J.A.D.Connolly, Y.Y.Podladchikov, Temperature-dependent viscoelastic compaction and compartmentalization in sedimentary basins, Tectonophysics, 324(2000), no. 3, 137-168.

[4] R.E.Grimm, S.C.Solomon, Viscous relaxation of impact crater relief on Venus: Constraints on crustal thickness and thermal gradient, Journal of Geophysical Research: Solid Earth, 93(1988), no. B10, 11911-11929.

[5] A.A.Papin, M.A.Tokareva, On Local solvability of the system of the equation of onedimensional motion of magma, Journal of Siberian Federal University. Mathematics and Physics, 10(2017) no. 3, 385-395. DOI: 10.17516/1997-1397-2017-10-3-385-395

[6] M.N. Koleva, L.G.Vulkov, Numerical analysis of one dimensional motion of magma without mass forces, Journal of Computational and Applied Mathematics, 366(2020), 112338.

DOI: $10.1016 /$ j.cam.2019.07.003

[7] M.A.Tokareva, A.A.Papin, Global solvability of a system of equations of one-dimensional motion of a viscous fluid in a deformable viscous porous medium, Journal of Applied and Industrial Mathematics, 13(2019), no. 2, 350-362. DOI: 10.33048/sibjim.2019.22.208

[8] M.Simpson, M.Spiegelman, M.I.Weinstein, Degenerate dispersive equations arising in the stady of magma dynamics, Nonlinearity, 20(2007), no. 1, 21-49.

[9] V.Y.Rudyak, O.B.Bocharov, A.V.Seryakov, Hierarchical sequence of models and deformation peculiarities of porous media saturated with fluids, Proceedings of the XLI Summer School-Conference Advanced Problems in Mechanics (APM-2013), 2013, 184-191.

[10] O.B.Bocharov, V.Y.Rudyak, A.V.Seryakov, Simplest deformation models of a fluidsaturated poroelastic medium, Journal of Mining Science, 50(2014), 235-248.

[11] A.M.Abourabia, K.M.Hassan, A.M.Morad, Analytical solutions of the magma equations for molten rocks in a granular matrix, Chaos, Solitons and Fractals, 42(2009), no. 2, 1170-1180.

[12] Y.Geng, L.Zhang, Bifurcations of traveling wave solutions for the magma equation, Applied Mathematics and computation, 217(2010), no. 4, 1741-1748.

[13] I.G.Akhmerova, A.A.Papin, Solvability of the boundary-value problem for equations of onedimensional motion of a two-phase mixture, Mathematical Notes, 96(2014), no. 2, 166-179.

[14] C.Morency et al., A numerical model for coupled fluid flow and matrix deformation with applications to disequilibrium compaction and delta stability, Journal of Geophysical Research: Solid Earth, 112(2007), no. B10.

[15] A.S.Saad, B.Saad, M.Saad, Numerical study of compositional compressible degenerate twophase flow in saturated-unsaturated heterogeneous porous media, Comput. Math. Appl., 71(2016), no. 2, 565-584. 
[16] S.N.Antontsev, A.V.Kazhikhov, V.N.Monakhov, Boundary value problems of the mechanics of inhomogeneous fluids, Nauka. Sib. branch, 1983 (in Russian).

[17] A.A.Papin, I.G.Akhmerova, Solvability of the system of equations of one-dimensional motion of a heat-conducting two-phase mixture, Mathematical Notes, 87(2010), no. 2, 230-243.

[18] P.I.Lizorkin, A course of differential and integral equations with additional chapters of analysis, Nauka, 1981 (in Russian).

[19] O.A.Ladyzhenskaya, V.A.Solonnikov, and N.N.Ural'tseva, Linear and Quasilinear Equations of Parabolic Type, Moscow, Nauka, 1967 (in Russian).

\title{
Фильтрация жидкости в неизотермической вязкой пористой среде
}

\author{
Александр А. Папин \\ Маргарита А. Токарева \\ Рудольф А. Вирц \\ Алтайский государственный университет \\ Барнаул, Российская Федерация
}

Аннотация. Для системы уравнений одномерного нестационарного движения жидкости в теплопроводной вязкой пористой среде доказана разрешимость начально-краевой задачи.

Ключевые слова: закон Дарси, пороупругость, фильтрация, разрешимость, теплопроводность. 